\title{
ROLE OF EM IN THE DIAGNOSIS OF SMALLPOX AND OTHER CAUSES OF RASH ILLNESS
}

\author{
Cynthia S. Goldsmith, Inger K. Damon, Yasuo Ichihashi, Scott Schmid, and Sherif R. Zaki
}

Division of Viral and Rickettsial Diseases, National Center for Infectious Diseases, Centersfor Disease Control and Prevention (CDC), Atlanta, Georgia 30333

The last case of naturally occurring smallpox was in Somalia in 1975, and the disease was declared irradicated in 1980 by the World Health Organization. Because of its potential for devastation if reintroduced into a mostly naive population, variola virus, the causative agent of smallpox, has been placed in Category A of a list of Critical Biological Agents for Public Health Preparedness. In preparation for a possible intentional release of variola virus, the laboratories at CDC have refined their methodologies for the rapid diagnosis of potential smallpox infections, including ruling out other causes, both infectious and non-infectious.

Historically, patients with variola virus infections presented with a febrile prodrome and classic smallpox lesions (deep-seated, firm, round, well-circumscribed vesicles or pustules). Lesions displayed a centrifugal distribution (greatest concentration of lesions on face and distal extremities) and were found in the same stage of development on any one part of the body [1]. However, these symptoms can be confused with those of rash illnesses caused by other etiologic agents of viral exanthems, including herpesviruses (varicella and herpes simplex), enteroviruses (including agents of hand, foot and mouth disease), and morbillivirus (measles), as well as other poxviruses (disseminated vaccinia or molluscum contagiosum). These agents must be included in the differential diagnosis of patients presenting with a rash illness.

For EM diagnostics, the negative staining technique is rapid and is an excellent screening tool in that it can differentiate among several agents, thereby guiding further investigative studies. For diagnosis of rash illness, negative staining of vesicular or pustular fluid is done by means of a direct touch method, or resuspension of vesicular fluid dried on a microscopic slide [2,3]. Large, easily recognizable orthopox virions, approximately $250 \times 300 \mathrm{~nm}$ in size, can be seen (see Fig. 1). Although some virions have the well-known brick shape, most particles are rounded at one or more corners. In contrast, another exanthem agent, herpesvirus, is distinguishable in negative stain preparations by having an icosahedral nucleocapsid(Fig. 2) that is sometimes surrounded by the viral envelope to give a Afried egg@ appearance.

Other confirmatory EM methodologies are also in place. In clinical cases for which ultrastructural preservation is not optional, making a morphologic diagnosis difficult, immunogold labeling techniques are also available (Fig. 3). When crusts are available from patients, these may be ground for negative stain preparations and/or prepared for thin section examination, using microwave-assisted rapid embedding techniques (see Fig. 4)

The laboratory diagnosis of patients with a papular rash requires a multifaceted approach, including the techniques of histopathology, polymerase chain reactions (PCR), serology, virus isolation, and immunohistochemistry. However, ultrastructural examination of vesicular fluid remains crucial because of its rapidity and its potential for differentiating among etiologic agents.

References: 
[1] hittp://www.cdc.gov/nip/smallpox/poster-protocol.pdfi

[2] J.H. Nakano, Diagnostic Procedures for Viral, Rickettsial and Chlamydial Infections, American Public Health Association, Washington DC (1979) 257.

[3] H.R. Gelderblom and P.R. Hazelton, Emerging Infectious Diseases 6 (2000) 434.

[4] S. Dales, Ultrastructure of Animal Viruses and Bacteriophages: AnAtlas, Academic Press, New York (1973) 109.

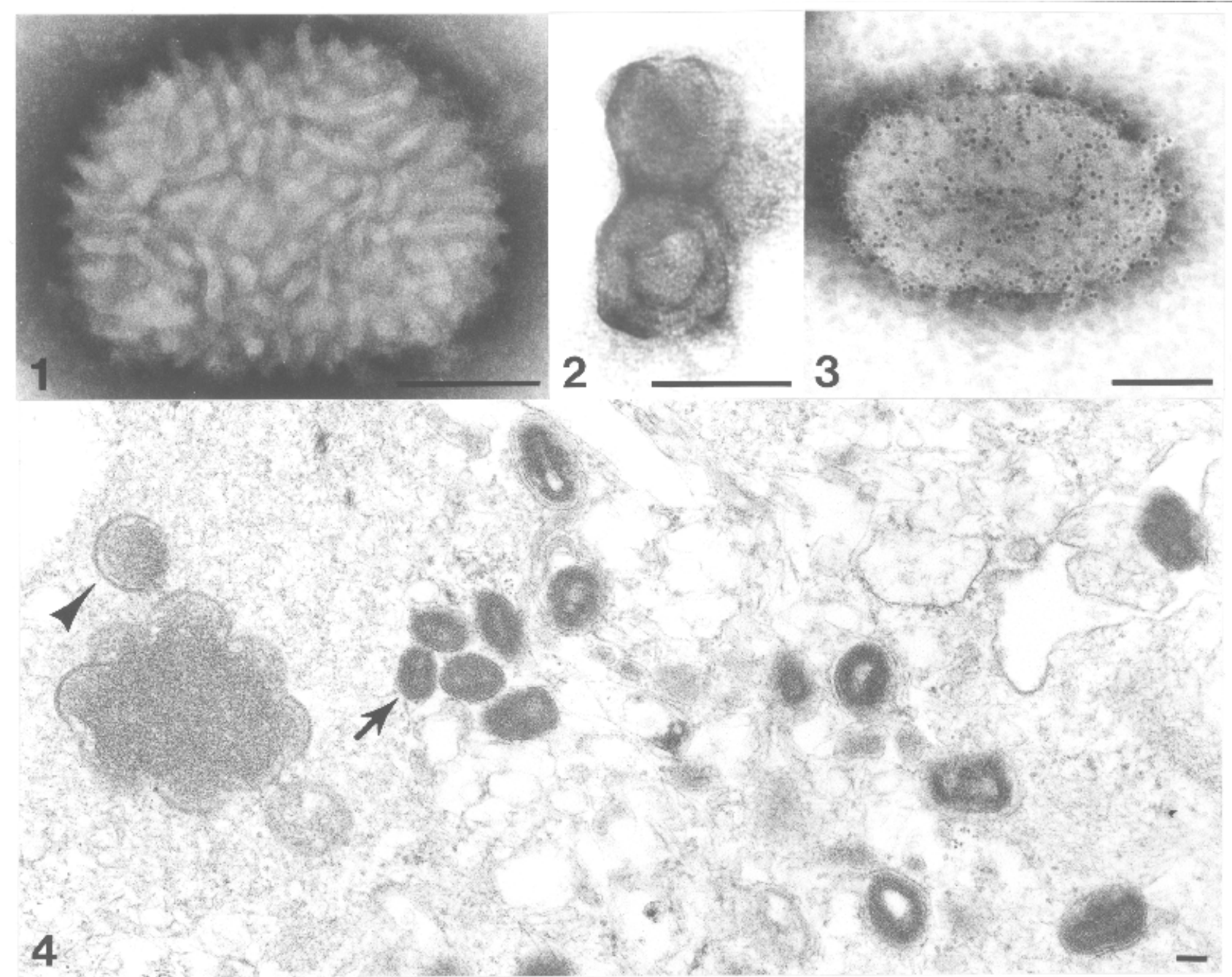

Figure legends. 1. Negative stain image of vaccinia virus isolate, the smallpox vaccine stain, grown in BSC-40 tissue culture cells. 2. Negative stain image of the herpesvirus varicella zoster, from a vesicula fluid specimen. In the lower particle, note the virus core within the icosahedral nucleocapsid. 3. Immunogold labeling of vaccinia virus isolate, using a hyperimmune mouse ascites fluid and $6 \mathrm{~nm}$ colloidal gold. 4. Variola virus isolate grown in BSC-40 tissue culture cells and embedded by standard techniques. Replication occurs in the cytoplasm of infected cells within virusAfactories,® or virosomes. Viroplasm is enclosed within spicules, to form spherical immature particles (arrowhead). These condense to form intracellular mature virions (arrow), which become enveloped at the Golgi apparatus, migrate to the cell surface, and are released [4]. Bars, $100 \mathrm{~nm}$. 